\title{
Correction to: Invited Discussion on: "Transaxillary Nipple- Sparing Mastectomy and Direct-to-Implant Breast Reconstruction Using a Simplified Endoscopic Approach: Indications, Cosmetic Outcomes and Technical Refinements",
}

\author{
Gianluca Campiglio ${ }^{1}$ (i)
}

Published online: 18 August 2020

(C) Springer Science+Business Media, LLC, part of Springer Nature and International Society of Aesthetic Plastic Surgery 2020

\section{Correction to: \\ Aesth Plast Surg \\ https://doi.org/10.1007/s00266-020-01877-x}

This erratum is to add reference citation to the original article discussed in this invited commentary. Original reference 1 in the article becomes reference 2 . Updated reference list appears below.

Unfortunately, it is still a common the idea that if an operation is aimed to treat a cancer, trauma or malformation it cannot be performed aesthetically at the same time, thus reducing its stigmata and lifelong cosmetic impact. The authors should be congratulated for submitting to this journal another successful example on how to overcome this ancestral conflict between reconstructive and aesthetic procedures that marked the last decades in plastic surgery [1]

Indeed, in the case of implant-based breast reconstruction more than in other fields of our specialty, two important innovations such as nipple sparing mastectomy (NSM) and prepectoral location of the prostheses greatly contributed to respond to this increasing demand for better aesthetic outcomes both from patients and surgeons.

The original article can be found online at https://doi.org/10.1007/ s00266-020-01877-x.

Gianluca Campiglio

info@gianlucacampiglio.it

1 Plastic Surgery Division, University of Milan, Via Tranquillo Cremona 12, 20145 Milan, Italy

\section{References}

1. Visconte $\mathrm{G}$ et al (2020) Transaxillary nipple-sparing mastectomy and direct-to-implant breast reconstruction using a simplified endoscopic approach: indications, cosmetic outcomes and technical refinements. Aesth Plast Surg. https://doi.org/10.1007/s00266-02001792-1

2. Campiglio GL, Candiani P (1995) Transaxillary submuscular augmentation mammaplasty. Eur J Plast Surg 18:108-110

Publisher's Note Springer Nature remains neutral with regard to jurisdictional claims in published maps and institutional affiliations. 